\title{
Bethe Ansatz and Geometry of the Classical Configuration Space
}

\author{
B. Lulek, T. LuLEK \\ Chair of Physics, Rzeszów University of Technology \\ Powstańców Warszawy 6, 35-959 Rzeszów, Poland \\ AND J. MILEWSKI \\ Institute of Mathematics, Poznań University of Technology \\ Piotrowo 3A, 60-965 Poznań, Poland
}

\begin{abstract}
We demonstrate that the seminal one-dimensional model of the Heisenberg magnet, consisting of $N$ spins $1 / 2$ with the nearest-neighbour isotropic interaction, solved exactly by Bethe ansatz, admits an interpretation of a system of $r=N / 2-M$ pseudoparticles (spin deviations) which are indistinguishable, have hard cores and move on the chain by local hoppings. Such an approach allows us to construct a manifold with some boundaries, which is genericly $r$-dimensional, and whose $F$-dimensional regions, $0<F<r$, point out all $l$-strings. The latter classify, in terms of rigged string configurations of Kerov, Kirillov and Reshetikhin, all exact Bethe eigenfunctions. In this way, we interpret these eigenfunctions in terms of the classical configuration space, in particular on the structure of islands of adjacent spin deviations, in a way independent of the size $N$.
\end{abstract}

PACS numbers: 02.10.Ox, 02.40.Sf, 31.15.xm, 75.75.+a, 85.75.Ff

\section{Introduction}

It is well known that the Heisenberg model of a magnetic ring, consisting of $N$ spins $1 / 2$ with the isotropic nearest-neighbour interaction, is the seminal example of a completely integrable system, so the corresponding Hamiltonian eigenproblem is solved exactly by the Bethe ansatz (BA) [1-3]. The analytic form of BA eigenfunctions is usually specified for a fixed number $r=N / 2-M$ spin deviations from the ferromagnetic saturation $|++\ldots+\rangle$, with $M$ being the magnetization, an exact quantum number resulting from the rotational symmetry. This fact allows one to interpret the corresponding Hilbert space $\mathcal{H}^{(r)}$ as that of a quantum system of $r$ Bethe pseudoparticles which move on the ring by hoppings between nearest neighbours. Here we aim to point out a classical counterpart of this quantum system along a general Schwinger scheme of unitary geometry for finite-dimensional Hilbert spaces [4-6]. In particular, we discuss here, in some detail, the geometry of the resulting classical configuration space for this system in the limit $N \rightarrow \infty$, with the fixed number $r$ of the Bethe pseudoparticles. We also indicate the way in which exact BA eigenfunctions, classified in terms of rigged string configurations along the Kerov-Kirillov-Reshetikhin (KKR) bijection [7-9], emerge from appropriate boundaries of the classical configuration space.

\section{The classical counterpart of the quantum system of $r$ Bethe pseudoparticles on the magnetic ring}

$$
\begin{aligned}
& \text { Let } \\
& \tilde{N}=\{j=1,2, \ldots, N\}
\end{aligned}
$$

be the set of nodes of the magnetic ring, such that $(j,(j+1) \bmod N)$ is a pair of nearest neighbours for $j \in \tilde{N}$, and

$$
\begin{aligned}
Q^{(r)} & =\left\{\boldsymbol{j}=\left(j_{1}, j_{2}, \ldots, j_{r}\right) \mid 1 \leq j_{1}<j_{2}<\ldots\right. \\
\left.<j_{r} \leq N\right\} &
\end{aligned}
$$

- the set of all magnetic configurations with $r$ spin deviations. Each vector $j \in Q^{(r)}$ is readily identified with one of possible positions of the system of $r$ Bethe pseudoparticles, and the set $Q^{(r)}$ exhausts each such position exactly once. Thus the quantum Hilbert space $\mathcal{H}^{(r)}$ of this system is the linear closure of this set over the field $\mathbb{C}$ of complex numbers, which we write as

$$
\mathcal{H}^{(r)}=l c_{\mathbb{C}} Q^{(r)}
$$

and treat the set $Q^{(r)}$ as an orthonormal basis of $\mathcal{H}^{(r)}$, i.e.

$$
\operatorname{dim} \mathcal{H}^{(r)}=\left|Q^{(r)}\right|=\left(\begin{array}{c}
N \\
r
\end{array}\right) .
$$

It allows us to identify the set $Q^{(r)}$, along the general picture of the Schrödinger quantisation, as the classical configuration space (i.e. the set of all admissible positions) for the quantum system

$$
\tilde{r}=\{\alpha=1,2, \ldots, r\}
$$


of the Bethe pseudoparticles. Moreover, the set $Q^{(r)}$ can be presented as [10]:

$$
Q^{(r)}=\left[\tilde{N}^{\times \tilde{r}} / D(N, r)\right] / \Sigma_{r},
$$

that is, as the generic stratum of the action of the symmetric group $\Sigma_{r}$ on the $r$-th Cartesian power $\tilde{N}^{\times \tilde{r}}$ of the set $\tilde{N}$, with $D(N, r)$ being the set of all coincidences of two or more pseudoparticles on the same node (the fat diagonal).

In other words, $Q^{(r)}$ acquires the status of the classical configuration space for the system $\tilde{r}$ of indistinguishable hard-core particles, with $\tilde{N}$ being the corresponding space for a single particle, $\Sigma_{r}$ playing the role of the Pauli indistinguishability group, and the fat diagonal $D(N, r)$ assuring that two spin deviations cannot occupy the same node.

\section{The geometry of the classical configuration space}

The identification of $Q^{(r)}$ as the classical configuration space, i.e. the set of all positions of the system, seems to be somehow formal until one imposes a notion of proximity between various points of this set. It can be readily done in two aspects: local and global one. The local aspect is determined in terms of the action of the Heisenberg Hamiltonian $H$ in the space $\mathcal{H}^{(r)}$, namely (cf., e.g. [10])

$$
\hat{H}|\boldsymbol{j}\rangle=\sum_{\boldsymbol{j}^{\prime} \in Q_{\boldsymbol{j}}^{(r)}}\left(\left|\boldsymbol{j}^{\prime}\right\rangle-|\boldsymbol{j}\rangle\right), \quad \boldsymbol{j} \in Q^{(r)},
$$

where $|\boldsymbol{j}\rangle \in \mathcal{H}^{(r)}$ is the quantum state corresponding to the magnetic configuration $j \in Q^{(r)}$, and $Q_{j}^{(r)}$ is the set of all such magnetic configurations $\boldsymbol{j}^{\prime} \in Q^{(r)}$ which differ from $|\boldsymbol{j}\rangle$ at exactly one component $j_{\alpha}, \alpha \in \tilde{r}$, and then $j_{\alpha}^{\prime}=\left(j_{\alpha} \pm 1\right) \bmod N$. In other words, $Q_{j}^{(r)}$ is the set of all nearest-neighbour magnetic configurations of $\boldsymbol{j}$ in $Q^{(r)}$, and the Hamiltonian acts locally.

It is clear that each Bethe pseudoparticle $\alpha \in \tilde{r}$ defines a spatial variable $j_{\alpha} \in \tilde{N}$, such that for $j$ in a general position, when

$$
j_{(\alpha \pm 1) \bmod r} \neq\left(j_{\alpha} \pm 1\right) \bmod N, \quad \alpha \in \tilde{r},
$$

that is, when none spin deviation is a nearest neighbour of the preceding or following one on the chain $\tilde{N}$, all variables $j_{\alpha}, \alpha \in \tilde{r}$, are independent. In other words, the classical configuration space $Q^{(r)}$ is genericly $r$-dimensional.

If, however, some Bethe pseudoparticles do not satisfy the condition (8), and thus form some islands of consecutive spin deviations on the chain $\tilde{N}$, then each such an island contributes just one independent spatial variable (since internal degrees of freedom are frozen by kinematical restrictions - pseudoparticles inside an island cannot move). Such cases constitute some boundaries of $Q^{(r)}$, with the local dimension $F$ equal to the total number of such islands. Clearly,

$$
1 \leq F \leq r
$$

and each value of $F$ within this range corresponds to some structures of islands of spin deviations on the classical configuration space $Q^{(r)}$.

The global aspect of the geometry of $Q^{(r)}$ is associated with the fact that $\tilde{N}$ is a ring. Formally, one introduces the translation operator $C_{N} \in \Sigma_{N}$ in $\mathcal{H}^{(r)}$ which acts on a magnetic configuration $\boldsymbol{j}=\left(j_{1}, j_{2}, \ldots, j_{r}\right)$ as

$$
C_{N}\left|j_{1}, j_{2}, \ldots, j_{r}\right\rangle=\mid\left\{\left(j_{1}+1\right) \bmod N, \ldots,\right.
$$

$$
\left.\left.\left(j_{r}+1\right) \bmod N\right\}\right\rangle, \quad j \in Q^{(r)},
$$

where the curly bracket indicates the periodic boundary conditions, so that, in particular, $\quad\left|\left\{\left(j_{1}+1\right) \bmod N, \ldots,(N+1) \bmod N\right\}\right\rangle \quad=$ $\left|1,\left(j_{1}+1\right) \bmod N, \ldots,\left(j_{r-1}+1\right) \bmod N\right\rangle$. It follows that each orbit $\mathcal{O}[\boldsymbol{j}]=\left\{\boldsymbol{j}, C_{N} \boldsymbol{j}, \ldots, C_{N}^{N-1} \boldsymbol{j}\right\} \subset Q^{(r)}$ should also constitute a ring, and thus all "Cartesian axes" $j_{\alpha}, \alpha \in \tilde{r}$, in $Q^{(r)}$ should be globally declined accordingly. In short, the translation group $C_{N}$ acts globally on the classical configuration space $Q^{(r)}$, its orbits form loops, imposing thus a nontrivial homotopy on it.

In order to discuss homotopy properties of the configuration space $Q^{(r)}$, we introduce a model manifold for it in the limit $N \rightarrow \infty$. Clearly, in this limit the $\operatorname{ring} \tilde{N}$ becomes a circle $S^{1}$ with the unit radius, which we denote as the topological factor space

$$
S^{1}=[0,2 \pi] /\{0,2 \pi\} \text {. }
$$

More formally, one says that each $\operatorname{ring} \tilde{N}, N=1,2, \ldots$, is embedded in $S^{1}$ by means of the mapping $i_{N}: \tilde{N} \rightarrow S^{1}$, given by $i_{N}(j)=2 \pi j / N, j \in \tilde{N}$. In the following, we refer to each mapping $i_{N}: \tilde{N} \rightarrow S^{1}$ as to an embedding, and to the target set $S^{1}$ - as to the model manifold for a ring $\tilde{N}$. Now, we need the corresponding model manifold $\mathcal{M}^{(r)}$ for a configuration space $Q^{(r)}$. We consider thus an infinite series of classical configuration spaces $Q^{(r)} \equiv Q^{(r)}(N)$ for fixed $r$ and $N \rightarrow \infty$. The associated embeddings $I_{N, r}: Q^{(r)}(N) \rightarrow \mathcal{M}^{(r)}$ are determined by

$$
I_{N, r}(\boldsymbol{j})=\boldsymbol{s} \in \mathcal{M}^{(r)}, \quad \boldsymbol{j} \in Q^{(r)}(N)
$$

with $\boldsymbol{j}=\left(j_{1}, \ldots, j_{r}\right), \boldsymbol{s}=\left(s_{1}, \ldots, s_{r}\right)$, and $s_{\alpha}=i_{N}\left(j_{\alpha}\right)$ for $\alpha \in \tilde{r}$. These embeddings yield the open-closed simplex

$$
\begin{aligned}
& \Delta^{(r)}=\left\{s \in \mathbb{R}^{r} \mid 0 \leq s_{1}<s_{2}<\ldots<s_{r} \leq 2 \pi,\right. \\
& \left.s_{r}-s_{1}<2 \pi\right\} \subset \mathbb{R}^{r}
\end{aligned}
$$

whose $r+1$ vertices are

$$
\begin{aligned}
& w_{0}=(0, \ldots, 0), w_{1}=(2 \pi, \ldots, 2 \pi), \\
& w_{2}=(0,2 \pi, \ldots, 2 \pi), \ldots, w_{r}=(0, \ldots, 0,2 \pi) .
\end{aligned}
$$

Faces related to sharp inequalities $s_{\alpha}<s_{(\alpha+1) \bmod r}$, $\alpha \in \tilde{r}$, in Eq. (13) correspond to interior of the manifold $\mathcal{M}^{(r)}$ and thus should be glued appropriately, whereas the smooth ones (i.e. $0 \leq s_{1}$ and $s_{r} \leq 2 \pi$ ) are related to its true boundaries. It follows that

$$
\mathcal{M}^{(r)}=\Delta^{r} / \sim
$$


Let $(N \mathbb{Z})^{r}$ and $\mathbb{Z}^{r}$ be two hypercubic lattices in $\mathbb{R}^{r}$ with the spacings $2 \pi / N$ and $2 \pi$ respectively, such that $(N \mathbb{Z})^{r} \subset \mathbb{Z}^{r} \subset \mathbb{R}^{r}$. Then $\mathbb{Z}^{r} /(N \mathbb{Z})^{r} \cong \tilde{N}^{\times \tilde{r}}$, and the simplex $\mathcal{M}^{(r)}$ can be seen as a piece of the elementary hypercube of the lattice $(N \mathbb{Z})^{r}$. Clearly,

$$
Q^{(r)}=\Delta^{(r)} \cap \mathbb{Z} / \sim
$$

i.e. the classical configuration space admits a local hypercubic lattice structure. Intersection with true frontiers of $\mathcal{M}^{(r)}$ yields fictitious configurations of BA, corresponding to coincidences of the Bethe pseudoparticles. Such frontiers constitute physical boundaries of the configuration space. It is worth to observe that the glued frontiers correspond to cyclic boundary conditions along Eq. (8), associated with relabelling the system $\tilde{r}$ when the last Bethe pseudoparticle $(\alpha=N)$ passes from the node $j=N$ to $j=1$, and thus becomes the first one. Such hyperfaces are not real boundaries of the classical configuration space but rather frontiers of the Cartesian map, and reflect homotopy properties of the model manifold $\mathcal{M}^{(r)}[11]$.

\section{Rigged string configurations}

We just sketch here that the famous Kerov-KirillovReshetikhin bijection [7] maps each magnetic configuration $\boldsymbol{j} \in Q^{(r)}$ to an exact BA eigenfunction $\operatorname{KKR}(\boldsymbol{j})=$ $\nu \mathcal{L}$, labelled by a rigged string configuration $\nu \mathcal{L}$, with $\nu \vdash r^{\prime}, 0 \leq r^{\prime} \leq r$ being a partition which reflects the composition of $l$-strings, and $\mathcal{L}$ denoting the set of quantum numbers called riggings, and reflecting the quasimomentum of each string. Within this context, an $l$-string, associated combinatoricly with the sequence of $2 l$ consecutive nodes $j+1, \ldots, j+l, j+l+1, \ldots, j+2 l$, the first $l$ occupied by the Bethe pseudoparticles, and the last $l$ by "vacuum"spins $+1 / 2$, has to originate from an island of spin deviations of the size not smaller than $l$. This observation yields a geography of strings on the classical configuration space.

\section{Conclusions}

Geometry of the classical configuration space $Q^{(r)}$ of the system of $r$ Bethe pseudoparticles can be described in terms of embedding $Q^{(r)} \subset \mathcal{M}^{(r)}$ into a simplex in $r$-dimensional real space, which thus becomes a model manifold for BA.

The Kerov-Kirillov-Reshetikhin bijection between all positions $j \in Q^{(r)}$ of the system and all exact BA eigenfunctions, labelled by rigged string configurations, associates each $l$-string with an island of the size $l$ (or larger) which constitutes an essential part of the corresponding wave packet. Such islands are located at specified boundaries of the model manifold $\mathcal{M}^{(r)}$.

\section{References}

[1] H. Bethe, Z. Phys. 71, 205 (1931) (in German; English translation in: D.C. Mattis, The Many-Body Problem, World Sci., Singapore 1993, p. 689).

[2] M. Gaudin, Le Fonction d'Onde de Bethe, Massons, Paris 1983.

[3] R.J. Baxter, Exactly Solvable Models in Statistical Mechanics, Academic Press, New York 1982.

[4] J. Schwinger, Quantum Kinematics and Dynamics, Benjamin, New York 1970.

[5] A. Vourdas, J. Phys. A, Math. Gen. 29, 4275 (1996).

[6] A. Vourdas, J. Phys. A, Math. Theor. 40, R285 (2007).

[7] S.V. Kerov, A.N. Kirillov, N.Yu. Reshetikhin, Leningradskoe Otdeleniye Matematicheskogo Instituta im. V.A. Steklova AN SSSR (LOMI) 155, 50 (1986) (in Russian; English translation: J. Sov. Math. 41, 916 (1988)).

[8] A. Schilling, MSJ Memoirs 17, 75 (2007).

[9] T. Lulek, B. Lulek, D. Jakubczyk, P. Jakubczyk, Physica B: Phys. Condens. Matter 382, 162 (2006).

[10] B. Lulek, T. Lulek, Rep. Math. Phys. 38, 267 (1996).

[11] J. Milewski, B. Lulek, T. Lulek, Phys. Status Solidi B 244, 2497 (2007). 